Supporting Information

\title{
ENHANCING HOST-CELL PROTEIN DETECTION IN PROTEIN THERAPEUTICS USING HILIC ENRICHMENT AND PROTEOMIC
}

\author{
ANALYSIS \\ Qingyi Wang ${ }^{1 *}$, Thomas R. Slaney ${ }^{2}$, Wei Wu ${ }^{2}$, Richard Ludwig ${ }^{2}$, Li Tao ${ }^{2}$, and Anthony \\ Leone $^{2}$ \\ ${ }^{1}$ Department of Chemistry, The University of Michigan, 930 North University Avenue, \\ Ann Arbor, Michigan 48109, United States \\ ${ }^{2}$ Biophysical Chemical Characterization Team, Bristol-Myers Squibb Company, 1 Squibb \\ Dr., New Brunswick, New Jersey 08901, United States \\ *qywang@umich.edu
}

\section{Table of Contents}

Table S1. Amounts of samples loaded onto the HILIC column for analysis ........................... S2

Figure S1. $280 \mathrm{~nm}$ UV profiles of HILIC separation of BMS mAb1 drug substance with HCP

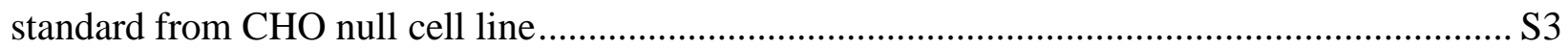

Table S2. LC mobile phase gradient and flow rate used in Figure S1 .................................. S3

Figure S2. 215 nm UV profiles of RP-LC separation of NISTmAb tryptic digests from the native digestion method vs. the HILIC enrichment method S4

Table S3. List of identified HCPs in NISTmAb (RM 8671) using the HILIC-enriched HCP LCMS method, with details S5

Table S4. Glycosylation details of the identified HCPs in NISTmAb by the native digestion method only S8

Figure S3. MS/MS spectra for the identified peptides of two newly-found HCPs from NISTmAb S10

Figure S4. $280 \mathrm{~nm}$ UV profile of HILIC separation of BMS fusion protein 1 S11 


\begin{tabular}{cccc}
\hline Sample names & $\begin{array}{c}\text { Mass of Therapeutic } \\
\text { Protein Injected }\end{array}$ & $\begin{array}{c}\text { Mass of HCP } \\
\text { Standard Injected }\end{array}$ & $\begin{array}{c}\text { Injection } \\
\text { Volume }\end{array}$ \\
\hline $\begin{array}{c}\text { BMS mAb1 with } \\
\text { concentrated HCP standard } \\
\text { BMS mAb1 with 1 } k \text { ppm } \\
\text { HCPs }\end{array}$ & $10 \mu \mathrm{g}$ & $9.4 \mu \mathrm{g}$ & $4 \mu \mathrm{L}$ \\
BMS mAb1 with 10k ppm & $2 \times 150 \mu \mathrm{g}^{*}$ & $2 \times 0.15 \mu \mathrm{g}^{*}$ & $4 \mu \mathrm{L}$ \\
HCPs & $2 \times 150 \mu \mathrm{g}^{*}$ & $2 \times 1.5 \mu \mathrm{g}^{*}$ & $4 \mu \mathrm{L}$ \\
$\begin{array}{c}\text { NISTmAb } \\
\text { BMS fusion protein 1 }\end{array}$ & $700 \mu \mathrm{g}$ & N/A & $250 \mu \mathrm{L}$ \\
\end{tabular}

Table S1. Amounts of samples loaded onto the HILIC column for analysis. For BMS mAb1 spiked with $1 k$ and $10 k$ HCP standard (marked by *), HILIC eluates from two consecutive runs were combined for fractions \#1, \#2, and \#3, respectively, prior to the drying/concentration step. 


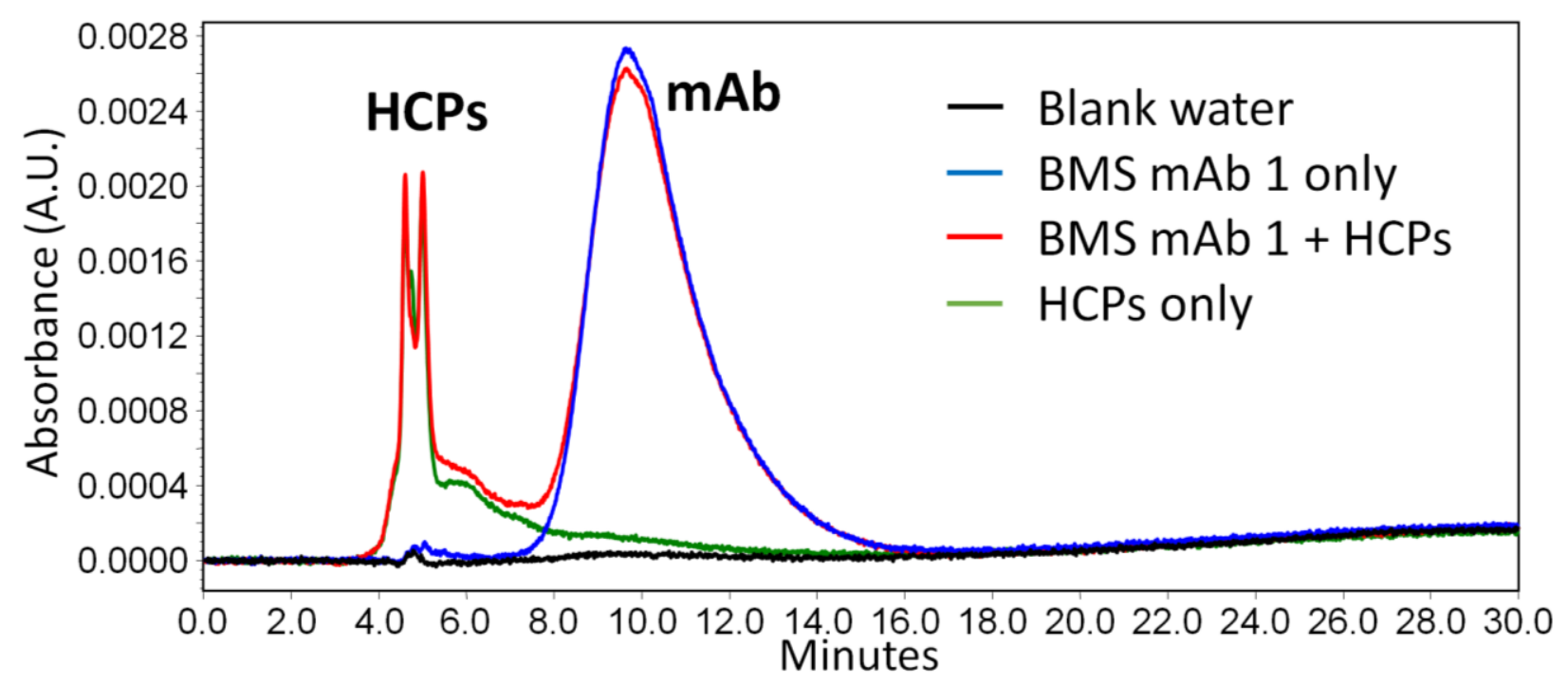

Figure S1. $280 \mathrm{~nm}$ UV profiles of HILIC separation of $5.0 \mu \mathrm{g}$ of BMS mAb1 drug substance with (red line) and without (blue line) addition of $4.7 \mu \mathrm{g}$ of HCP standard from CHO null cell line. Blank water (black line) and HCP standard only (green line) are also shown for reference. Waters XBridge HILIC column $(5 \mu \mathrm{m}, 4.6 \times 150 \mathrm{~mm})$ was used at an operating temperature of $45^{\circ} \mathrm{C}$. The mobile phase A was $20 \mathrm{mM}$ ammonium formate in water containing $0.1 \%$ formic acid and the mobile phase B was $20 \mathrm{mM}$ ammonium formate in 90/10 acetonitrile/water containing $0.1 \%$ formic acid.

\begin{tabular}{cccc}
\hline Time (min) & $\begin{array}{c}\text { Flow rate } \\
(\mathbf{m L} / \mathbf{m i n})\end{array}$ & $\mathbf{A \%}$ & $\mathbf{B \%}$ \\
\hline 0 & 0.4 & 50.0 & 50.0 \\
25 & 0.4 & 99.0 & 1.0 \\
30 & 0.4 & 99.0 & 1.0 \\
31 & 0.4 & 50.0 & 50.0 \\
34 & 0.6 & 50.0 & 50.0 \\
36 & 0.6 & 99.5 & 0.5 \\
39 & 0.6 & 99.5 & 0.5 \\
41 & 0.6 & 50.0 & 50.0 \\
61 & 0.4 & 50.0 & 50.0 \\
62 & 0.4 & 50.0 & 50.0 \\
\hline
\end{tabular}

Table S2. LC mobile phase gradient and flow rate used in Figure S1. 

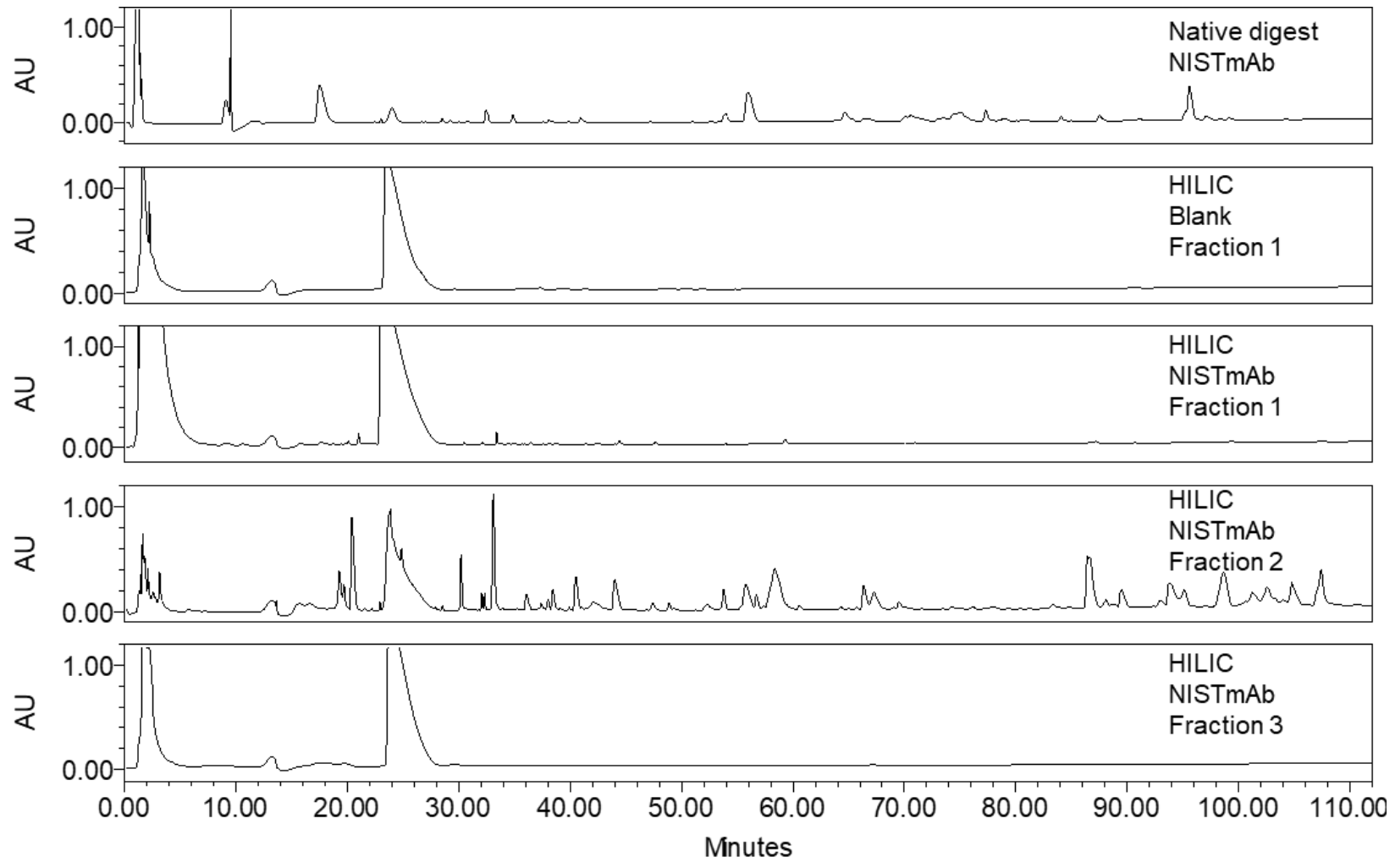

Figure S2. $215 \mathrm{~nm}$ UV peptide map chromatograms of NISTmAb tryptic digests for the native digestion method, the blank control of HILIC (injecting water only) Fraction \#1, the HILIC Fraction \#1, the HILIC Fraction \#2, and the HILIC Fraction \#3, respectively (from top to bottom). Peptide peaks visible in native digest and Fraction \#2 are likely from digested NISTmAb, demonstrating the level of depletion per sample. 


\begin{tabular}{|c|c|c|c|c|c|c|}
\hline No. & Accession \# & HCP name & \# of unique peptides & Coverage \% & Fraction \# & Glycosylation \\
\hline 1 & P05064 & Fructose-bisphosphate aldolase A & 32 & 63.46 & $1,2,3$ & \\
\hline 2 & P05063 & Fructose-bisphosphate aldolase C & 26 & 52.62 & 1 & \\
\hline 3 & P06745 & Glucose-6-phosphate isomerase & 31 & 49.1 & 1 & \\
\hline 4 & Q9CZ44 & NSFL1 cofactor $\mathrm{p} 47$ & 14 & 37.84 & 1 & \\
\hline 5 & Q922R8 & Protein disulfide-isomerase A6 & 11 & 15 & 1 & \\
\hline 6 & O88569 & Heterogeneous nuclear ribonucleoproteins A2/B1 & 10 & 25.5 & 1 & \\
\hline 7 & Q5SUH7 & Clathrin interactor 1 & 7 & 12.52 & 1 & \\
\hline 8 & Q9WTP6 & Adenylate kinase 2 , mitochondrial & 11 & 43.51 & 1 & \\
\hline 9 & Q9ER00 & Syntaxin-12 OS = Mus musculus & 6 & 24.45 & 1 & \\
\hline 10 & Q68FL6 & Methionine--tRNA ligase, cytoplasmic & 3 & 4.66 & 1 & \\
\hline 11 & P56812 & Programmed cell death protein 5 & 4 & 38.89 & 1 & \\
\hline 12 & Q923D2 & Flavin reductase (NADPH) & 5 & 33.98 & 1 & \\
\hline 13 & Q8K4Z5 & Splicing factor $3 \mathrm{~A}$ subunit 1 & 6 & 8.22 & 1 & \\
\hline 14 & Q8K019 & Bcl-2-associated transcription factor 1 & 7 & 5.44 & 1 & \\
\hline 15 & F6ZDS4 & Nucleoprotein TPR & 5 & 2.3 & 1 & \\
\hline 16 & Q91YR9 & Prostaglandin reductase 1 & 5 & 13.98 & 1 & \\
\hline 17 & P49312 & Heterogeneous nuclear ribonucleoprotein A1 & 4 & 15.94 & 1 & \\
\hline 18 & P40142 & Transketolase & 4 & 10.43 & 1 & \\
\hline 19 & P62984 & Ubiquitin-60S ribosomal protein $\mathrm{L40}$ & 4 & 34.37 & 1 & \\
\hline 20 & Q9DBP5 & UMP-CMP kinase & 4 & 23.47 & 1 & \\
\hline 21 & P45878 & Peptidyl-prolyl cis-trans isomerase FKBP2 & 4 & 32.86 & 1 & \\
\hline 22 & Q8BGD9 & Eukaryotic translation initiation factor $4 \mathrm{~B}$ & 3 & 6.87 & 1 & \\
\hline 23 & Q99PL5 & Ribosome-binding protein 1 & 2 & 1.87 & 1 & \\
\hline 24 & $\mathrm{P} 21460$ & Cystatin-C & 4 & 22.86 & 1 & \\
\hline 25 & Q9QUR8 & Semaphorin-7A & 3 & 7.08 & 3 & $5 \mathrm{~N}$-linked \\
\hline 26 & Q8BL97 & Serine/arginine-rich splicing factor 7 & 6 & 24.72 & 1 & \\
\hline 27 & Q68FF6 & ARF GTPase-activating protein GIT1 & 6 & 6.62 & 1 & \\
\hline 28 & Q9Z0X1 & Apoptosis-inducing factor 1 , mitochondrial & 4 & 10.29 & 1 & \\
\hline
\end{tabular}




\begin{tabular}{|c|c|c|c|c|c|c|}
\hline No. & Accession \# & HCP name & \# of unique peptides & Coverage \% & Fraction \# & Glycosylation \\
\hline 29 & O08583 & THO complex subunit 4 & 2 & 14.12 & 1 & \\
\hline 30 & Q8K4F5 & Protein ABHD11 & 5 & 18.24 & 1 & \\
\hline 31 & 035143 & ATPase inhibitor, mitochondrial & 6 & 27.36 & 1 & \\
\hline 32 & Q9D8B3 & Charged multivesicular body protein $4 \mathrm{~b}$ & 4 & 16.96 & 1 & \\
\hline 33 & Q9CXW3 & Calcyclin-binding protein & 3 & 9.61 & 1 & \\
\hline 34 & Q60864 & Stress-induced phosphoprotein 1 & 4 & 6.81 & 1 & \\
\hline 35 & Q03173 & Protein enabled homolog & 6 & 9.35 & 1 & \\
\hline 36 & P55302 & $\alpha$-2-macroglobulin receptor-associated protein & 4 & 11.67 & 1 & $1 \mathrm{~N}$-linked \\
\hline 37 & P01887 & $\beta$-2-microglobulin & 7 & 26.05 & 1 & \\
\hline 38 & Q8CGC7 & Bifunctional glutamate/proline--tRNA ligase & 2 & 2.31 & 1 & \\
\hline 39 & P70372 & ELAV-like protein 1 & 2 & 11.04 & 1 & \\
\hline 40 & P70333 & Heterogeneous nuclear ribonucleoprotein $\mathrm{H} 2$ & 2 & 4.9 & 1 & \\
\hline 41 & Q99020 & Heterogeneous nuclear ribonucleoprotein A/B & 3 & 12.63 & 1 & \\
\hline 42 & Q9CQE1 & Protein NipSnap homolog 3B & 4 & 17.41 & 1 & \\
\hline 43 & Q9D2M8 & Ubiquitin-conjugating enzyme E2 variant 2 & 5 & 32.41 & 1 & \\
\hline 44 & Q61335 & B-cell receptor-associated protein 31 & 4 & 11.43 & 1 & \\
\hline 45 & P97855 & Ras GTPase-activating protein-binding protein 1 & 5 & 12.26 & 1 & \\
\hline 46 & Q3TLH4 & Protein PRRC2C & 2 & 1.05 & 1 & \\
\hline 47 & Q9DB15 & $39 \mathrm{~S}$ ribosomal protein $\mathrm{L} 12$, mitochondrial & 3 & 15.92 & 1 & \\
\hline 48 & P08249 & Malate dehydrogenase, mitochondrial & 3 & 10.65 & 1 & 1 O-linked \\
\hline 49 & P99029 & Peroxiredoxin-5, mitochondrial & 2 & 8.57 & 1 & \\
\hline 50 & P10126 & Elongation factor $1-\alpha 1$ & 2 & 4.33 & 1 & \\
\hline 51 & 035166 & Golgi SNAP receptor complex member 2 & 2 & 10.85 & 1 & \\
\hline 52 & Q922I7 & MCG13402, isoform CRA_c & 4 & 5.77 & 1 & \\
\hline 53 & P23116 & Eukaryotic translation initiation factor 3 subunit $\mathrm{A}$ & 4 & 2.23 & 1 & \\
\hline 54 & Q61035 & Histidine--tRNA ligase, cytoplasmic & 2 & 3.93 & $\mathbf{1}$ & \\
\hline 55 & P62897 & Cytochrome c, somatic & 1 & 10.48 & 1 & \\
\hline 56 & Q62504 & Msx2-interacting protein & 3 & 0.96 & 1 & \\
\hline
\end{tabular}




\begin{tabular}{|c|c|c|c|c|c|c|}
\hline No. & Accession \# & HCP name & \# of unique peptides & Coverage \% & Fraction \# & Glycosylation \\
\hline 57 & P40124 & Adenylyl cyclase-associated protein 1 & 3 & 5.49 & 1 & \\
\hline 58 & Q99K48 & Non-POU domain-containing octamer-binding protein & 3 & 5.92 & 1 & \\
\hline 59 & P62843 & 40S ribosomal protein S15 & 1 & 8.97 & 1 & \\
\hline 60 & Q62418 & Drebrin-like protein & 2 & 4.59 & 1 & \\
\hline 61 & Q8CI11 & Guanine nucleotide-binding protein-like 3 & 3 & 4.46 & 1 & \\
\hline 62 & P54227 & Stathmin & 3 & 14.77 & 1 & \\
\hline 63 & 008784 & Treacle protein & 1 & 1.06 & 1 & \\
\hline 64 & D3Z0M9 & DEAD (Asp-Glu-Ala-Asp) box polypeptide 23 & 3 & 3.42 & $\mathbf{1}$ & \\
\hline 65 & Q9CQF3 & Cleavage and polyadenylation specificity factor subunit 5 & 1 & 5.29 & 1 & \\
\hline 66 & Q3U0P5 & Ectonucleoside triphosphate diphosphohydrolase 6 & 1 & 2.2 & 1 & \\
\hline 67 & P51954 & Serine/threonine-protein kinase Nek1 & 3 & 1.5 & 1 & \\
\hline 68 & Q9JIX8 & Apoptotic chromatin condensation inducer in the nucleus & 4 & 5.16 & 1 & \\
\hline 69 & A2AFI3 & RNA binding motif protein, $X$ chromosome, isoform CRA_b & 1 & 4.32 & 1 & \\
\hline 70 & Q9DBG7 & Signal recognition particle receptor subunit $\alpha$ & 1 & 1.89 & 1 & \\
\hline 71 & Q62179 & Semaphorin-4B & 1 & 1.22 & 3 & $9 \mathrm{~N}$-linked \\
\hline
\end{tabular}

Table S3. Identified HCPs in NISTmAb (RM 8671) using the HILIC-enriched HCP LCMS method. For each identified HCP, the number of unique peptides observed, protein sequence coverage \%, which HILIC fraction it came from, and its glycosylation profile are also listed. Accession numbers are for the Uniprot database. For each HCP, \# of unique peptides is the total number of peptide-to-spectrum matches (PSMs) discounting duplicates; coverage \% is the percent of the protein sequence covered by PSMs. Glycosylation information was obtained from the Uniprot database. Newly identified HCPs in NISTmAb using the HILIC-enriched HCP method, which are not observed using the native digestion method, are highlighted in bold. 


\begin{tabular}{|c|c|c|c|}
\hline No. & Accession \# & HCP Name & $\begin{array}{c}\text { Number of } \\
\text { N-linked } \\
\text { Glycosylation } \\
\text { Sites }\end{array}$ \\
\hline 1 & Q9EPX2 & Papilin & 0 \\
\hline 2 & Q8C7U7 & Polypeptide N-acetylgalactosaminyltransferase 6 & 2 \\
\hline 3 & P26928 & Hepatocyte growth factor-like protein & 4 \\
\hline 4 & P08101 & Low affinity immunoglobulin gamma $\mathrm{Fc}$ region receptor II & 4 \\
\hline 5 & P17892 & Pancreatic lipase-related protein 2 & 2 \\
\hline 6 & Q3UEB3 & Poly(U)-binding-splicing factor PUF60 & 0 \\
\hline 7 & P60335 & Poly(rC)-binding protein 1 & 0 \\
\hline 8 & Q6ZWZ6 & $40 \mathrm{~S}$ ribosomal protein $\mathrm{S} 12$ & 0 \\
\hline 9 & Q9D7P6 & Iron-sulfur cluster assembly enzyme ISCU, mitochondrial & 0 \\
\hline 10 & O88967 & ATP-dependent zinc metalloprotease YME1L1 & 0 \\
\hline 11 & E9PZF0 & Nucleoside diphosphate kinase & 0 \\
\hline 12 & P03975 & IgE-binding protein & 0 \\
\hline 13 & P35700 & Peroxiredoxin-1 & 0 \\
\hline 14 & Q8BJD1 & Inter- $\alpha$-trypsin inhibitor heavy chain $\mathrm{H} 5$ & 8 \\
\hline 15 & Q8R0W0 & Epiplakin & 0 \\
\hline 16 & Q9D1M4 & Eukaryotic translation elongation factor 1 epsilon- 1 & 0 \\
\hline 17 & P02798 & Metallothionein-2 & 0 \\
\hline 18 & O35737 & Heterogeneous nuclear ribonucleoprotein $\mathrm{H}$ & 0 \\
\hline 19 & P32020 & Non-specific lipid-transfer protein & 0 \\
\hline 20 & Q6PB93 & Polypeptide $\mathrm{N}$-acetylgalactosaminyltransferase 2 & 1 \\
\hline 21 & Q9JIX0 & Transcription and mRNA export factor ENY2 & 0 \\
\hline 22 & Q62165 & Dystroglycan & 4 \\
\hline 23 & O08807 & Peroxiredoxin-4 & 0 \\
\hline 24 & P28798 & Granulins & 4 \\
\hline 25 & Q9Z1D1 & Eukaryotic translation initiation factor 3 subunit $\mathrm{G}$ & 0 \\
\hline 26 & Q03402 & Cysteine-rich secretory protein 3 & 3 \\
\hline 27 & P97807 & Fumarate hydratase, mitochondrial & 0 \\
\hline 28 & P06151 & L-lactate dehydrogenase A chain & 0 \\
\hline 29 & P46425 & Glutathione S-transferase P 2 & 0 \\
\hline 30 & P70349 & Histidine triad nucleotide-binding protein 1 & 0 \\
\hline 31 & Q9D706 & RNA polymerase II-associated protein 3 & 0 \\
\hline 32 & P09411 & Phosphoglycerate kinase 1 & 0 \\
\hline 33 & Q9WVA4 & Transgelin-2 & 0 \\
\hline 34 & P45591 & Cofilin-2 & 0 \\
\hline 35 & O70305 & Ataxin-2 & 0 \\
\hline 36 & P30681 & High mobility group protein $\mathrm{B} 2$ & 0 \\
\hline 37 & Q61937 & Nucleophosmin & 0 \\
\hline
\end{tabular}




\begin{tabular}{c|ccc}
\hline No. & Accession \# & HCP Name & $\begin{array}{c}\text { Number of } \\
\text { N-linked }\end{array}$ \\
& & & $\begin{array}{c}\text { Glycosylation } \\
\text { Sites }\end{array}$ \\
\hline 38 & Q8BND5 & Sulfhydryl oxidase 1 & 2 \\
39 & Q64287 & Interferon regulatory factor 4 & 0 \\
40 & J3QNT7 & Epsin-2 & 0 \\
41 & Q9WV55 & Vesicle-associated membrane protein-associated protein A & 0 \\
42 & P56542 & Deoxyribonuclease-2- $\alpha$ & 4 \\
43 & Q6ZWY6 & Ubiquitin-conjugating enzyme E2 D2B & 0 \\
\hline
\end{tabular}

Table S4. HCPs identified in NISTmAb by the native digestion method that were not observed by the HILIC-enriched method. Glycosylation information was obtained from the Uniprot database. 

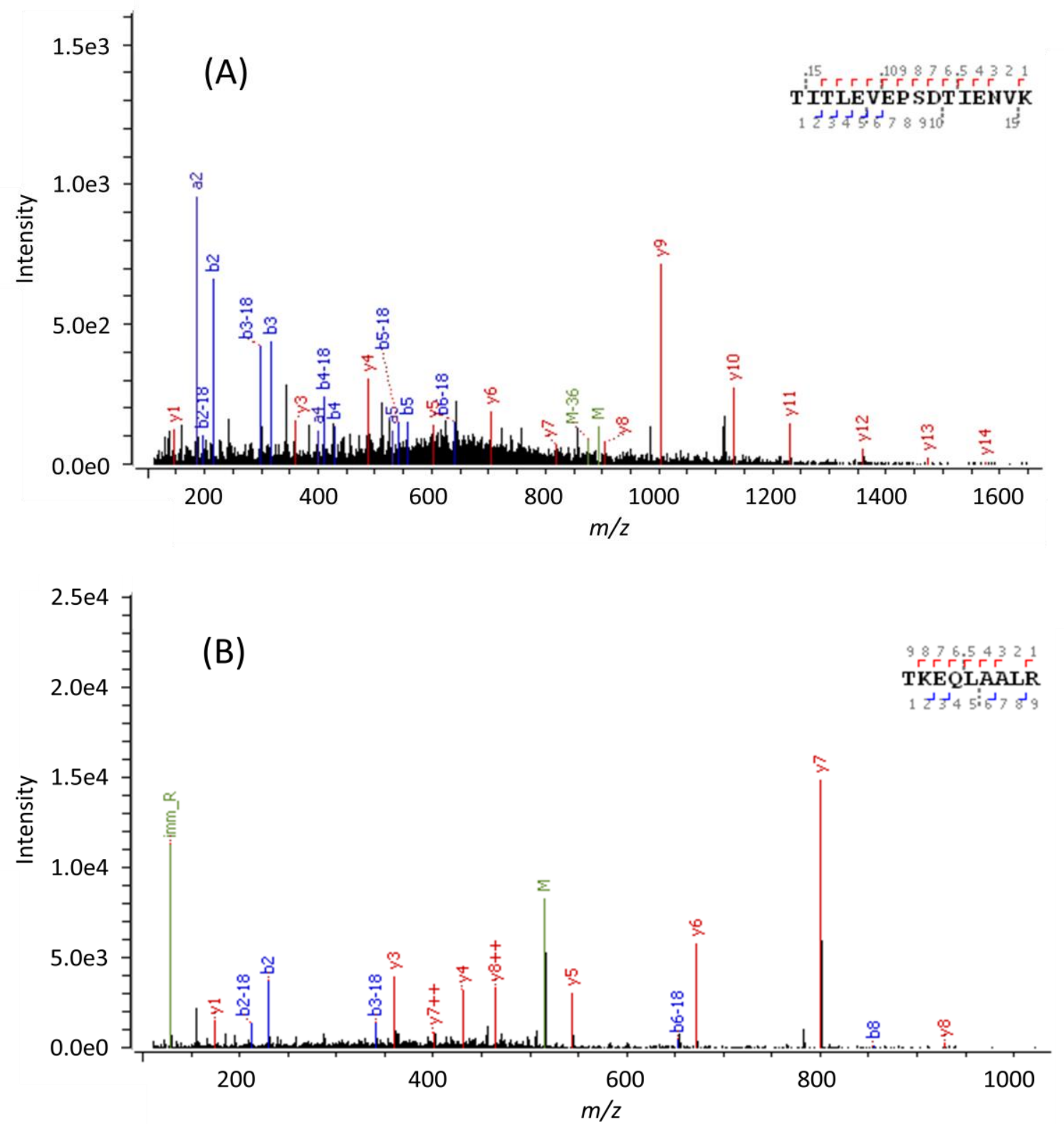

Figure S3. MS/MS representative spectra for peptides of two newly-found HCPs from NISTmAb, (A) ubiquitin-60S ribosomal protein L40 (accession no. P62984) and (B) ATPase inhibitor, mitochondrial (accession no. O35143). 


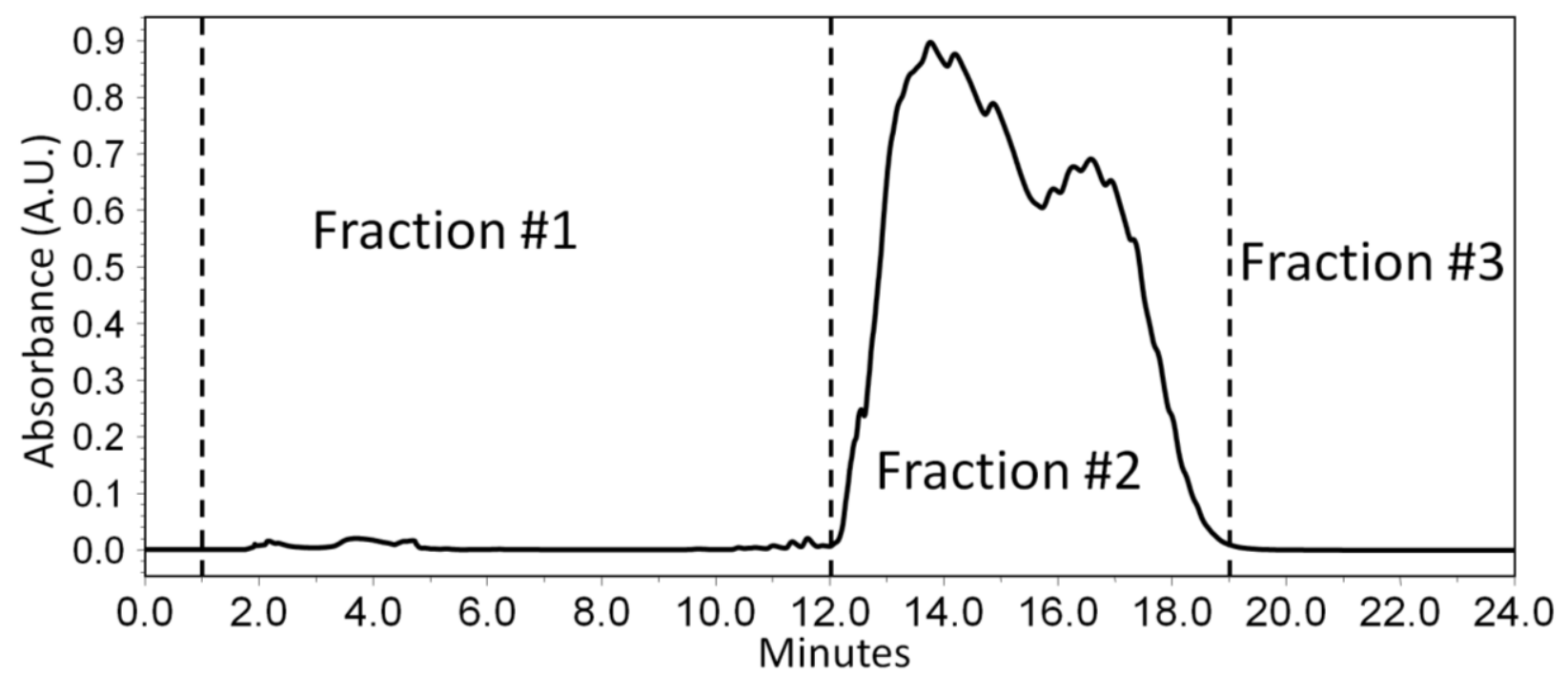

Figure S4. $280 \mathrm{~nm}$ UV chromatogram of HILIC separation of $700 \mu \mathrm{g}$ of BMS fusion protein 1. Retention time windows were 1.0-12.0 min for Fraction \#1, 12.0-19.0 min for Fraction \#2, and 19.0-24.0 min for Fraction \#3. 